Draft Version OCtOBER 19, 2018

Preprint typeset using $\mathrm{L}^{A} \mathrm{~T}_{\mathrm{E}} \mathrm{X}$ style emulateapj v. 01/23/15

\title{
MERGER HISTORY OF CENTRAL GALAXIES IN SEMI-ANALYTIC MODELS OF GALAXY FORMATION
}

\author{
Mojtaba Raouf,${ }^{1}$ Habib G. Khosroshahi, ${ }^{1,2}$ Gary A. Mamon, ${ }^{2}$ Darren J. Croton, ${ }^{3}$ Abdolhosein Hashemizadeh, ${ }^{4}$ \\ Ali A. DARIUSH ${ }^{5}$ \\ ${ }^{1}$ School of Astronomy, Institute for Research in Fundamental Sciences (IPM), Tehran, 19395-5746, Iran \\ ${ }^{2}$ Institut d'Astrophysique de Paris (UMR 7095: CNRS \& Sorbonne Université), 98 bis Bd. Arago, F-75014 Paris, France \\ ${ }^{3}$ Centre for Astrophysics \& Supercomputing, Swinburne University of Technology, PO Box 218, Hawthorn, Victoria 3122, Australia \\ ${ }^{4}$ International Centre for Radio Astronomy Research (ICRAR), The University of Western Australia, 35 Stirling Highway, Crawley, WA \\ 6009, Australia and \\ ${ }^{5}$ Institute of Astronomy, University of Cambridge, Madingley Road, Cambridge CB3 0HA, UK \\ Draft version October 19, 2018
}

\section{ABSTRACT}

We investigate the dynamical evolution of galaxies in groups with different formation epochs. Galaxy groups have been selected to be in different dynamical states, namely dynamically old and dynamically young, which reflect their early and late formation times, respectively, based on their halo mass assembly. Brightest galaxies in dynamically young groups have suffered their last major galaxy merger typically $\sim 2$ Gyr more recently than their counterparts in dynamically old groups. Furthermore, we study the evolution of velocity dispersion in these two classes and compare them with the analytic models of isolated halos. The velocity dispersion of dwarf galaxies in high mass, dynamically young groups increases slowly in time, while the analogous dispersion in dynamically old high-mass groups is constant. In contrast, the velocity dispersion of giant galaxies in low mass groups decreases rapidly at late times. This increasing velocity bias is caused by dynamical friction, and starts much earlier in the dynamically old groups. The recent RADIO-SAGE model of galaxy formation suggests that radio luminosities of central galaxies, considered to be tracers of AGN activity, are enhanced in halos that assembled more recently, independent of the time since the last major merger.

Subject headings: galaxies: groups : evolution - galaxies: formation - galaxies: general - galaxies: individual (old, young) - galaxies: structure

\section{INTRODUCTION}

In the hierarchical structure formation framework, massive galaxy systems, such as clusters, are formed through the mergers of smaller mass systems, such as galaxy groups. This halo growth is expected to have consequences on the constituent galaxies. In an isolated halo, dynamical friction (Chandrasekhar 1943) should cause galaxy orbits to decay toward the center of groups over a time-scale of a few Gyr (Jones, Ponman \& Forbes 2000 Tollet et al. 2017). As a result, given sufficient time, the galaxies within the core of the halo, e.g. $r<$ $0.5 r_{\text {vir }}$, would merge and form a significantly more luminous (Ostriker \& Tremaine 1975) and massive galaxy, leaving a substantial luminosity gap between the brightest group galaxy (BGG) and the second brightest.

Such systems, dubbed as fossil groups, have been identified in simulations, where it was shown that the luminosity gap is a simple indicator of the the frequency of galaxy mergers (Schneider \& Gunn 1982, Mamon 1987). Moreover, by analyzing the evolution of the halo mass of fossil groups in cosmological dark matter simulations, D'Onghia et al. (2005) showed that more than $50 \%$ of the present-epoch halo mass of fossil groups assembled at $z \gtrsim 1$, through growth by minor mergers. Following this pioneering study, Dariush et al. (2007) showed that a luminosity gap of $\sim 2$ magnitudes at the current epoch would mean a $\sim 25 \%$ more massive progenitor at $z=1$, than average. Other studies have looked for more efficient ways of identifying early formed halos. Sales et al. (2007) and Dariush et al. (2010) investigated the lu-

*m.raouf@ipm.ir minosity gap between the first, 4th and 10th luminous galaxy in the group. Raouf et al. (2014) took this a step further and showed that a combination of the luminosity gap and a measure of the halo relaxation, probed by the off-set between the BGG and the group luminosity centroid, is even more efficient in distinguishing the early and late formed halos.

The rate of galaxy mergers in groups and clusters is known to scale as $1 / \sigma_{v}^{3}$, where $\sigma_{v}$ is the group/cluster velocity dispersion, both in direct mergers (Mamon 1992 Makino \& Hut 1997, Krivitsky \& Kontorovich 1997), and as a result of dynamical friction (e.g., Jiang et al. 2008) ${ }^{1}$ This suggests that the low velocity dispersion is the driving parameter for building large magnitude gaps. Since the circular velocity of haloes in $\Lambda \mathrm{CDM}$ simulations is roughly constant from 7 Gyr ago $(z \sim 1)$ (e.g. Mamon et al. 2012, Tweed et al. 2018), one expects that the observable redshift evolution of group velocity dispersions can be used to test effects of mergers in groups of different dynamical ages. In the present article, we track the redshift evolution of velocity dispersion for galaxy groups of different dynamical ages and compare with the expected analogous evolution of the dark matter particles in their parent halos.

Other studies have added detail to this picture. Von Benda-Beckmann et al. (2008) presented evidence for the connection between a large luminosity gap in fossil groups and the early infall of massive satellites due to

1 The dynamical friction merger rate between subhaloes was calibrated with hydrodynamical simulations by Jiang et al. (2008), to yield a rate proportional to $\ln \left(1+M_{1} / M_{2}\right) /\left(M_{1} / M_{2}\right)$, which is roughly proportional to $1 / M_{1}$, hence to $\sigma_{v}^{-3}$. 
their earlier halo formation with respect to the normal groups. They found that the time since the last major merger for a majority of fossils was over 3 Gyr. Recently, Kundert, D'Onghia \& Aguerri (2017) showed that large luminosity gap $\left(\Delta M_{12} \geqslant 2\right) z=0$ systems assembled most of their mass before $z \sim 0.4$ (4 Gyr ago) and have a lack of recent mass accretion, in comparison to groups with $\Delta M_{12}<2$.

More generally, the properties of a BGG may be related to its luminosity gap and thus highlight differences in the group assembly history. For exmple, BGGs that sit in the cores of large luminosity gap groups/clusters tend to have non-boxy isophotes (Khosroshahi et al. 2006a, Smith et al. 2010), compared to those of similar mass in small luminosity gap groups/clusters. However, there are no significant differences in the stellar populations of BGGs in observed groups with large and small luminosity gaps (La Barbera et al. 2009; Trevisan, Mamon \& Khosroshahi 2017). Moreover, analyzing hydro-simulations, Cui et al. (2011) found no trend of luminosity gap with age, metallicity, colour, concentration or mass-to-light ratio. They also found that the number density of satellite galaxies in fossils are similar to those in normal groups. They concluded that fossils are transient phases in the evolution of ordinary galaxy groups.

Recently, Khosroshahi et al. (2017) discovered a significant difference in the AGN activity of the BGGs probed by their radio emission at $1.4 \mathrm{GHz}$. They found that the BGGs in dynamically relaxed groups (old) are less radio luminous compared to the BGGs of similar mass residing in dynamically unrelaxed groups (young). This suggests that AGN activity in BGGs is influenced by the dynamics of the group. This follows earlier clues from studies of a limited sample of confirmed fossil galaxy groups, which indicated a relatively low radio emission associated with the fossil BGGs (Jetha et al. 2008; Miraghaei et al. 2014, 2015). Accretion onto the super-massive black hole in a galaxy that has experienced its last major merger a few Gyr earlier is expected to be lower due to the lack of significant new mergers or tidal effects, itself caused by the absence of massive neighboring galaxies in large luminosity gap systems. However this does not rule out other forms of accretion, such as hot mode accretion. That said, the epoch of the last major merger may determine whether radio emission is boosted in dynamically young galaxy groups. By exploiting the Illustris hydrodynamical simulation, we showed that the central supermassive black hole in an old group is more massive than one in a young group, and that black hole accretion occurs at a slower rate (Raouf, Khosroshahi \& Dariush 2016).

Fossil groups have also been scrutinized by other means such by their halo concentration (Khosroshahi et al. 2004a Khosroshahi, Ponman, \& Jones 2007, Buote 2016) and X-ray properties (Jones et al. 2003, Khosroshahi et al. 2006b). Significant steps have been taken to understand the nature of fossil groups (Aguerri et al. 2011), although sample selection and a possible contamination in the samples have resulted in some contradictions. For instance, Kundert, D'Onghia \& Aguerri (2017) found no difference in the group mass formation at redshift $\sim 1$ due to a conservative sample selection for comparison of fossil $\left(\Delta M_{12} \geqslant 2\right)$ and non-fossil $\left(\Delta M_{12}<2\right)$ groups.

Cosmological simulations have helped us understand
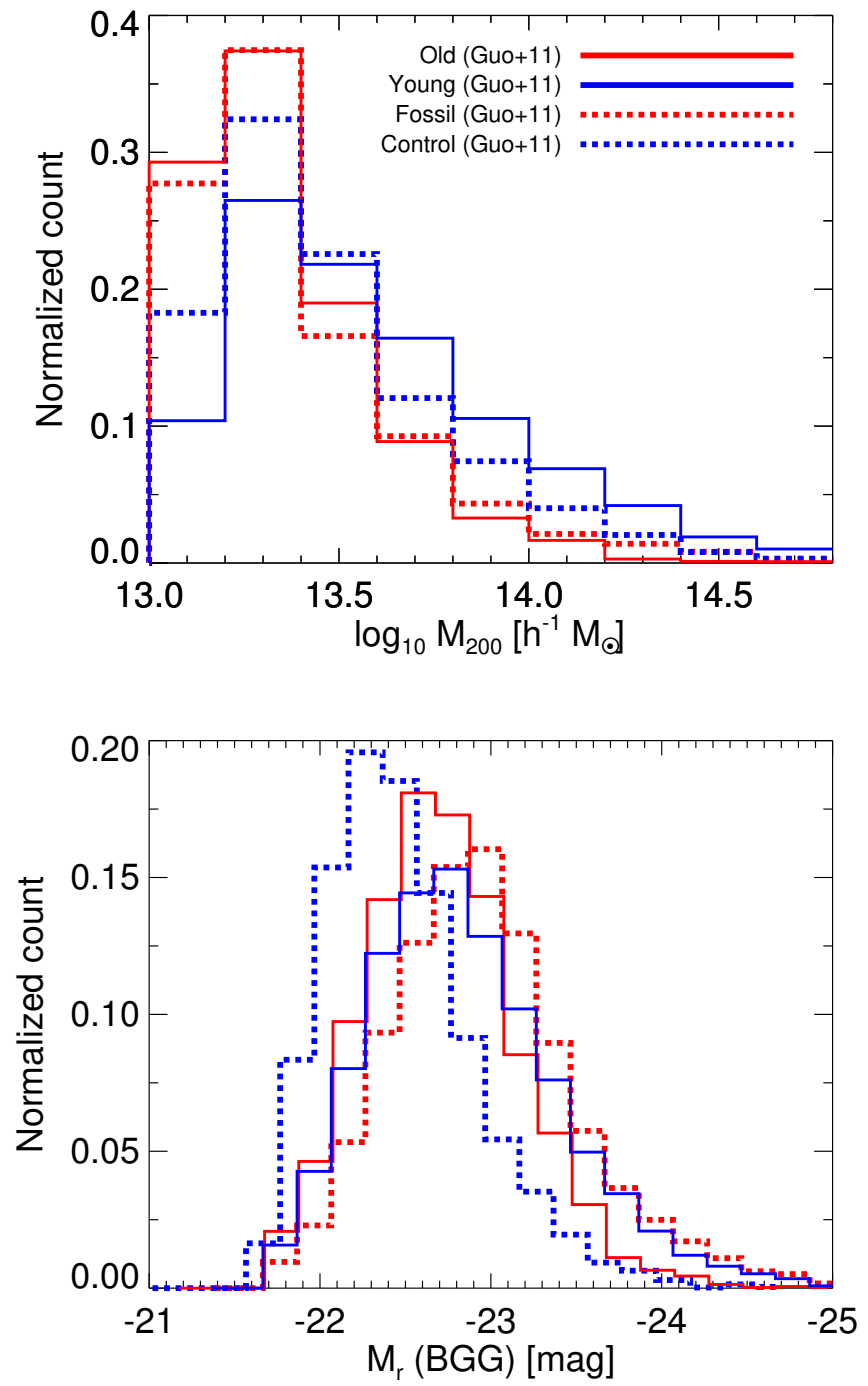

FIG. 1. - Distributions of $z=0$ halo mass (top) and BGG r-band absolute magnitude (bottom) for dynamically old (red line), young (blue line), fossil (red dashed line) and control (blue dashed line) galaxy groups identified in the Guo et al. (2011) semi-analytic model.

the halo-galaxy connection, while observations of galaxy properties in groups, and in particular where a distinction is made according to dynamical state, have allowed us to probe the success of galaxy formation models, implemented semi-analytically or hydro-dynamically (Raouf et al. 2014; Raouf, Khosroshahi \& Dariush 2016, Gozaliasl et al. 2014, 2016). In this tradition, here we revisit the merger history of the dominant galaxy. We study the impact of the last major merger of central galaxies within dynamically old and young groups of galaxies on their associated $1.4 \mathrm{GHz}$ AGN radio emission, as predicted by the RADIO-SAGE semi-analytic model of galaxy formation. We take a closer look at galaxy velocity dispersions in groups and its evolution, and compare it with analytical predictions.

Today's large-scale cosmological dark matter simulations, coupled with Semi-Analytical Models (SAMs) of galaxy formation, have made it possible to track the properties of groups and clusters of galaxies backward in 

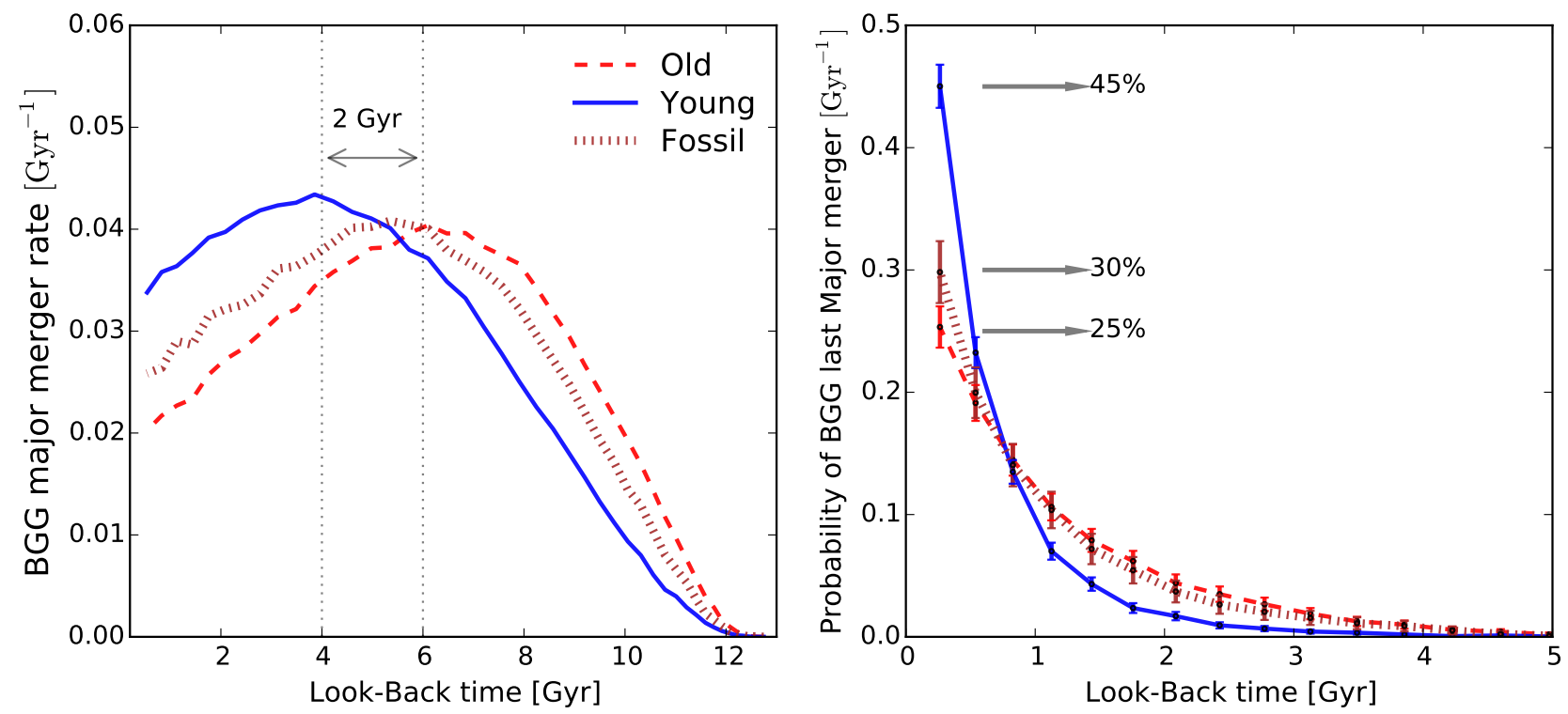

FIG. 2.- Left: Merger history of the BGG in three categories of groups: dynamically old (red long-dashed line) and young (blue line) groups of galaxies along the fossil $\left(\Delta M_{12}>2\right)$ groups (brown dotted line), for halo masses above $10^{13} h^{-1} \mathrm{M}_{\odot}$. Major mergers are characterized by mergers with $m_{1} / m_{2} \leq 3$. As seen, the BGGs of old systems shows a higher probability for a major merger at earlier epochs compared with young and fossil systems (about 2 Gyr in peak). Right: Comparison of the distributions of time since the last major merger between the three group categories. The error bars are Poisson. The last BGG merger in young groups is a more recent event than the same for the BGGs in fossil and old groups, respectively.

time and study their evolutionary histories. In this way, we can measure and trace back the physical properties of galaxies, which is otherwise impossible to do observationally. We utilize the Millennium Simulation (Springel et al. 2005), one of the largest cosmological dark matter simulations ever performed, together with the semianalytical galaxy catalogues of Guo et al. (2011) and our RADIO-SAGE model (Raouf et al. 2017). This allows us to identify and study fossil and control groups of galaxies, as well as old and young galaxy systems representing the relaxed and unrelaxed galaxy groups, as introduced in Raouf et al. (2014).

The structure of the paper is as follows: the data and sample selections are described in Section2. In Section 3 , we present our results and analysis. Finally, we provide a summary and discussion in Section 4. Throughout this paper, we adopt $H_{0}=100 h \mathrm{~km} \mathrm{~s}^{-1} \mathrm{Mpc}^{-1}$ for the Hubble constant with $h=0.73$.

\section{DATA AND SAMPLE SELECTION}

\subsection{Millennium Simulation}

In this study, we used the Millennium Simulation (hereafter, MS, Springel et al. 2005), containing $2160^{3}$ particles with a mass resolution of $8.6 \times 10^{8} h^{-1} \mathrm{M}_{\odot}$ within the box size of $\left(500 h^{-1} \mathrm{Mpc}\right)^{3}$. The simulation begins at redshift $z=127$ and evolves up to the present epoch and stores the data in 64 separate snapshots. The epochs of interest to us are $z<1$, taking into account the importance of the last $\sim 7 \mathrm{Gyr}$ in the evolution of galaxy groups, as well as the limitations of the simulation in terms of the halo mass and a number of group members. The MS assumed cosmology parameters as described in Wilkinson Microwave Anisotropy Probe -1 (WMAP-1; Komatsu et al. 2003). Dark matter halos were found by means of a friends-of-friends (FoF) algo- rithm as described in Davis et al. (1985). The SUBFIND algorithm (Springel 2001) was then applied to the FoF catalog in order to identify subhalos by restricting boundaries of substructures. The halo mass resolution in the MS is 20 particles, i.e. a minimum halo mass of $1.72 \times 10^{10} h^{-1} \mathrm{M}_{\odot}$.

\subsection{Semi-Analytical Models}

Halo merger trees provided in the publicly available German Astrophysical Virtual Observatory (GAVO) database 2 allow us to link halos and sub-halos between time-steps (snapshots). Semi-Analytical Models (SAMs) follow the many branches of the merger tree from past to present with specific recipes for galaxy properties, such as the gas, and stars of the bulge and disk components of galaxies (Croton et al. 2006, Bower et al. 2006, De Lucia \& Blaizot 2007; Guo et al. 2011; Raouf et al. 2017). In this study, we use the publicly available semi-analytical catalogue of Guo et al. (2011), which is successful in predicting the observed luminosity and stellar mass functions of galaxies, from the SDSS data and recent determinations of the abundance of satellite galaxies around the Milky Way as well as the clustering properties of galaxies as a function of stellar mass by updating the number of physical processes such as galaxy morphology and environmental effects and the treatments of the transition between the central and satellite galaxies during merger. Our sample includes $\sim 39000$ halos of mass $M_{200}>10^{13} h^{-1} \mathrm{M}_{\odot}$ with at least four member galaxies brighter than $M_{r}=-14$ at $z=0$. In Sections 3.1, 3.2, and 3.3, we use this semi-analytical model to study the merger history, the evolution of the group velocity dispersion (traced by its galaxies) and of the radial distribution of group members.

\footnotetext{
2 http://gavo.mpa-garching.mpg.de/MyMillennium
} 
For comparing the activity of central supermassive black holes in Section 3.4, we use a new galaxy formation model which is a modified version of the SAGE semianalytic model (Croton et al. 2016) run on the Millennium simulation (Springel et al. 2005), which we hereafter refer to as RADIO-SAGE (Raouf et al. 2017) ${ }^{3}$ RADIO-SAGE incorporates a new method for tracing the physical properties of radio jets in massive galaxies, including the evolution of radio lobes and their impact on the surrounding gas. In our model, we self-consistently trace the cooling-heating cycle that significantly shapes the life and death of many types of galaxies. We compute the radio luminosity, which is an important observable quantity to study AGN, through the radio luminosity function, the calibration of the jet power and the environmental effects on AGN. For self-consistency, we first updated the hot halo gas density profile into which the jets propagate. This affects the hot gas cooling rates into the galaxy. We make predictions for intermittent triggering and radio emission from AGN jets and construct the radio luminosity function at the same time as the usual stellar mass functions. The use of two semianalytic models in this study is driven by data products available from these simulations. The details of the Guo et al. (2011) SAM are not in conflict with those of RADIOSAGE within the scope studied here.

\subsection{Sample Selections}

A useful parameter used in this study, as a proxy for halo mass assembly, is the ratio of the halo mass at any given redshift $z$ to its final mass at $z=0$, i.e. $\alpha_{z, 0} \equiv M_{z} / M_{z=0}$. In our earlier study it was argued that a sample of fossil groups identified based on the luminosity gap, $\Delta M_{12}$, will result in a contaminated sample if the objective was to form a sample of early formed halos. However, by adding a constraint on the separation between the group luminosity weight and the position of its BGG, $D_{\text {off }}$, we considerably improved the age-dating method for classifying relaxed and unrelaxed galaxy groups and clusters, in comparison to the method based on the luminosity gap only (Raouf et al. 2014). There are other studies suggesting alternative definitions for early formed (relaxed) systems based on the magnitude gap. Accordingly, Dariush et al. (2010) found 50\% more early formed systems adopting a magnitude gap definition of $\Delta M_{14} \geq 2.5 \mathrm{mag}$ as opposed to the conventional criterion of $\Delta M_{12} \geq 2.0 \mathrm{mag}$.

In this work, samples of fossil/control and old/young groups of galaxies are selected from both the SAM group catalogs of Guo et al. (2011) and the RADIO-SAGE (dynamically old and young) model according to the following criteria:

(i) Fossil/Control systems: Fossil groups are defined with the conventional definition of a large magnitude gap, $\Delta M_{12} \geq 2.0 \mathrm{mag}$ (Jones et al. 2003). We use the $r$-band absolute magnitude to estimate the gap within $0.5 R_{200}$ of halos, where $R_{200}$ is the radius in which the mean mass density is 200 times the critical density, $\rho_{\mathrm{c}}(z)$. Observationally, fossil groups have a minimum bolometric X-ray luminosity of $L_{\mathrm{x}, \mathrm{bol}} \geq 0.25 \times 10^{42} h^{-2} \mathrm{erg} \mathrm{s}^{-1}$, which

3 https://github.com/mojtabaraouf/sage according to the Millennium gas simulation, almost corresponds to groups with halo masses of $M\left(R_{200}\right) \gtrsim 10^{13} h^{-1} \mathrm{M}_{\odot}$ (Dariush et al. 2007). Furthermore, fossil groups possess a giant elliptical galaxy at the center of their halos. To take this into account, our sample of fossil groups are limited to those having BGG absolute $r$-band magnitudes of $M_{r}(\mathrm{BGG}) \leq-21.5 \mathrm{mag}$. In addition, when comparing fossils with normal groups, we define control groups of galaxies as systems with $\Delta M_{12}<0.5 \mathrm{mag}$ but with the same halo mass and BGG luminosity as in fossil systems. Based on this criteria, we find 6938 ( 18\%) fossil and $6053(15 \%)$ control groups in the Guo et al. (2011) SAM.

(ii) Old/young systems: According to Raouf et al. (2014), old galaxy systems have assembled more than $50 \%$ of their final halo mass by $z \approx 1.0$, young galaxy systems have only acquired less than $30 \%$ of their final halo masses by $z=1$. Considering these constraints, together with the same limits on BGG magnitudes and halo masses as applied to select fossil/control systems, we find $12678(\sim 32 \%)$ old and $7478(\sim 19 \%)$ young groups in the Guo et al. (2011) SAM. We identified the same fraction of old and young systems in the RADIO-SAGE group catalogue. We find that $\sim 54 \%$ of fossil groups overlap with old systems and only $\sim 37 \%$ of control groups are also young systems.

Figure 1 shows the distributions of halo mass and BGG $r$-band luminosities of our selected samples at $z=0$ in the Guo et al. 2011) SAM.

\section{ANALYSIS AND RESULTS}

\section{1. $B G G$ merger history}

The formation of giant elliptical galaxies through major mergers of disk galaxies have been shown in both simulations and observations (Toomre \& Toomre 1972 Naab et al. 2009). Based on this merger scenario, large galaxies lose angular momentum due to dynamical friction and fall into the center of groups where they coalesce to form giant elliptical galaxies (White 1976, Schneider \& Gunn 1982; Mamon 1987; Ponman et al. 1994) that are frequently observed at the center of fossil groups and clusters of galaxies. In a scenario presented by Barnes (1989), the BGGs of fossil groups, known to be among the most massive galaxies in the Universe, are formed through the merging of central bright galaxies in compact galaxy groups. According to this scenario, compact groups are suggested to be the progenitors of fossil groups and central giant elliptical galaxies are the final remnants of group scale mergers. Using N-body simulations, Farhang et al. (2017) traced the halo mass of compact groups from $z=0$ to $z=1$ and showed that, on average, $55 \%$ of the halo mass in compact groups is assembled since $z \sim 1$, compared to $40 \%$ of the halo mass in fossil groups over the same time interval. This indicates that, compared to fossil groups, compact groups are relatively younger galaxy systems. They found that while $\sim 25 \%$ of fossil groups go through a compact phase, most fail to meet the compact group isolation criterion, leaving only $\sim 30 \%$ of fossil groups fully satisfying the compact group selection criteria. 
Some studies have found no sign of a recent major merger in the most luminous galaxy within some fossil groups. For instance, Khosroshahi et al. (2006a) and Smith et al. (2010) have pointed out the absence of ongoing group-group mergers and of major galaxy-galaxy mergers in fossil systems, based on X-ray studies of the morphology of a sample of regular/relaxed fossil groups. Such a result directly points to an early formation epoch in fossil groups.

The age-dating method used in this study is based on the mass assembly history of group halos, as introduced by Raouf et al. (2014). It is interesting to see how the BGG merger history is influenced by the mass assembly of its host halo. To this end, and by using the merger trees of the MS and tracing the histories of the BGGs, we study the merger history of the BGGs in three types of groups i.e., the old, young and fossil galaxy groups as described in Section 2.3.

We only consider groups with halo masses $\geq$ $10^{13} h^{-1} \mathrm{M}_{\odot}$. We classify mergers into major and minor based on the mass ratio $m_{1} / m_{2}$ of galaxies involved in merging. A merger is categorized as major or minor if $m_{1} / m_{2} \leq 3$ or $m_{1} / m_{2}>3$, respectively. Figure 2 shows probability distributions of this major merger rate (left-panel) and the time of the last major merger (rightpanel) of the central dominant galaxy, estimated at different epochs in old, young and fossil groups. The recent study of Kundert, D'Onghia \& Aguerri (2017) showed that large gap $\left(\Delta M_{12} \geqslant 2\right)$ groups assembled less than $20 \%$ of their mass recently (between $z=0$ and 0.4 ), in comparison to normal gap $\left(\Delta M_{12}<2\right)$ groups. As can be seen in the left panel, the major merger phenomenon in BGGs in old (already like fossil) groups peaks at an earlier epoch $(\approx 2.0$ Gyr $)$ compared to young groups. This indicates that the evolutionary history of the halos is somehow dictated by mergers. The right-panel of Figure 2 suggests that $45 \%$ of young systems experience their last major merger before the look-back time of $\sim$ $0.3 \mathrm{Gyr}$, while the same in old systems is $25 \%$. In addition, more than $45 \%$ of old systems have met their last major merger at look-back times $\geq 1.0 \mathrm{Gyr}$, in comparison with only about $15 \%$ for young systems. Note that, the predictions for galaxy merger rates can differ significantly among theoretical methods (see e.g. Hopkins et al. 2010 Rodriguez-Gomez et al. 2015, Guo \& White 2008).

\subsection{Redshift evolution of velocity dispersion}

Not only do galaxy mergers lead to larger magnitude gaps, but they may affect the velocity dispersions of groups. Dynamical friction causes the dissipation of orbital energy of galaxies, which depends on their location in the viral sphere and the group to galaxy mass ratio (see footnote 1). In high-mass groups, the dynamical friction time scales should be too long to reduce the velocity dispersion in the last 7.6 Gyr (i.e. since $z=1$ ), even for massive galaxies. In contrast, in low mass groups, dynamical friction should lead to short orbital decay times for intermediate- and high-mass galaxies.

In Figure 3, we study the evolution of line-of-sight velocities dispersion (1D velocity dispersion) in dwarf, intermediate and giant members of old, young, fossil and control groups of galaxies, for different group mass range.

The figure shows that velocity dispersion in young and control groups, and for all members of massive systems, increases with time (panels: b1, b2, b3, c1, c2 and c3), suggesting that these groups are unrelaxed systems. This increase in group velocity dispersion in young and control systems may be caused by the high fraction of major mergers of galaxies within these groups at redshifts $z<1$ (Figure 2).

While there no noticeable changes in the trend of young and control subsamples velocity dispersion through their evolution with time in the low mass groups of galaxies (panels: a1, a2), we can see a slight decrease in the velocity dispersion of giant members (panel: a3). In contrast, there are no significant variations in the evolution of velocity dispersion of old and fossil galaxy groups, suggesting that these groups are relaxed systems, except perhaps for the giant members of low and intermediate mass systems (panels: a3 and b3) where the velocity dispersion decreases with time due to dynamical friction.

In general, Figure 3 shows a wide range of velocity dispersion fluctuations through redshift in both unrelaxed and relaxed systems. These findings also show the hierarchical evolution of galaxy groups through their period of life where young groups, which indeed are nonvirialized, grow up in size and mass, leading to an increase of galaxy velocity dispersion in such systems. In contrast, old groups are systems which have been formed following the virialization of young systems in a hierarchical way, and that their halo dynamics/masses have not experienced a noticeable change for a long period of time ${ }^{4}$ Like fossil groups, the velocity dispersion of old groups shows a flat trend from $z \approx 1$ to $z=0$, while young and control groups display important evolution in their velocity dispersion. We note that less than $4 \%$ of the groups in our samples have less than or equal to two giant members, which would result in a velocity dispersion which is not useful in practice. The error bars show the small effects of the sampling noise on the estimated velocity dispersions.

\subsection{Radial Distribution of Galaxy Group Members}

In Figure 4, we show the radial distribution of galaxy group members in fossil/control and old/young groups as a function of their radial distance to the BGG for various halo mass bins. The stellar density follows the NFW model (Navarro, Frenk \& White 1996) up to the virial radius and becomes steeper at larger radii, because of how the galaxies are assigned to their haloes. The upper panels indicate that the control groups have higher stellar mass density in the inner regions than fossil groups. However, within the same radius and compared to young groups, the higher density of galaxies in old systems implies are similarly centrally concentrated.

Similar density distributions are shown for giant galaxies defined as those with $M_{r} \leq-21.5$. By comparing the mass density of giant galaxies in old groups and at different radius with those in fossil groups, we find that the abundance of giant galaxies on the outskirts of old groups is less than around fossil groups. In addition, a majority of giant galaxies, which mostly represent the

4 Figure 7 in Dariush et al. (2007) shows mass assembly of a typical fossil group and a control group from the Millennium gas simulation from redshift $z=1$ to 0 . While the fossil group has already largely been assembled, the control group has considerable substructure even at a later epoch 

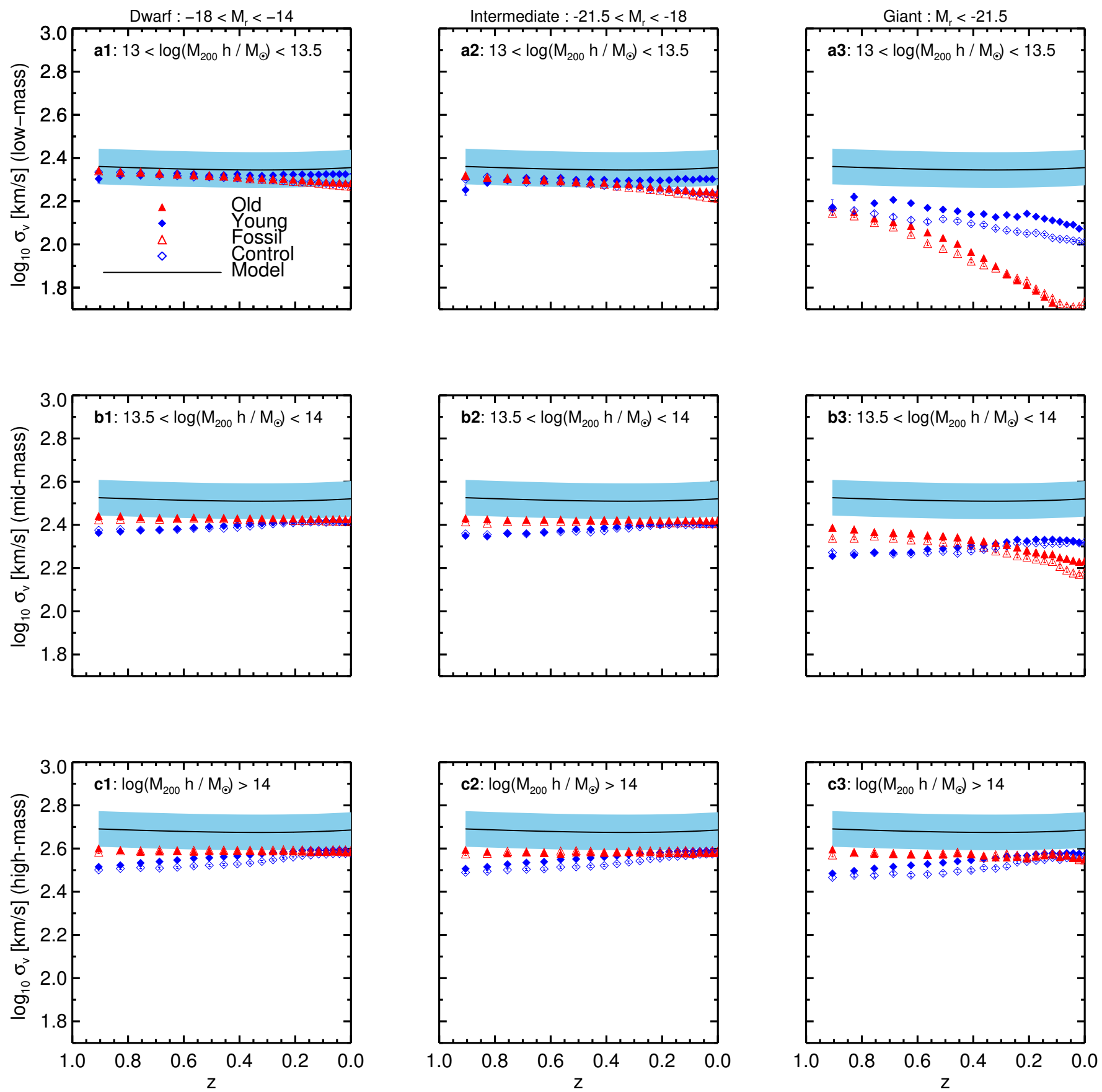

FIG. 3.- Redshift evolution of the 1D group velocity dispersion measured within $R_{200}$ using the dwarf, intermediate and giant galaxies (left, middle and right columns, respectively) for old (red filled triangles) and young (blue filled diamonds) groups, as well as fossil (red triangles) and control (blue diamonds) groups. The symbols show the mean values, and the error bars are the uncertainties on the mean $(\sigma / \sqrt{N})$. The variation of mean velocity dispersion for the model of isolated halos with assuming $f_{c} \equiv \sigma_{v} / V_{v}=0.65$ (see Appendix A , are displayed as black curves and the blue shaded regions indicate the upper and lower limits with typical 0.25 dex uncertainties for the $z=0$ halo mass range. Groups are split into three mass bins from top to bottom.

BGG counterparts in old groups, settle at the centers of halos within $r \leq 0.2 \mathrm{Mpc} / h$. A gap also exists between fossil and control systems around $0.2 \leq r \leq 0.5 \mathrm{Mpc} / h$ (top panels in Figure 4). This is due to our selection based on the magnitude gap. Similarly, there is a peak at radii larger than $r \geq 0.5 \mathrm{Mpc} / h$ in the distribution of giant galaxies in fossil groups, again showing a selection effect in finding relaxed systems based on the magnitude gap only criterion, due to the lack of $M_{*}$ galaxies in fos- sil groups. We also note that the BGGs are at the very center in all panels of the above figure and do not appear in these plots. Such selection effects are not observed in genuine old groups as giant galaxies in such systems have had enough time to fall towards the center of their host halos through dynamical friction (Von Benda-Beckmann et al. 2008). The expected NFW model for such a distribution is shown with the black dashed-line in all panels of the above figure. 

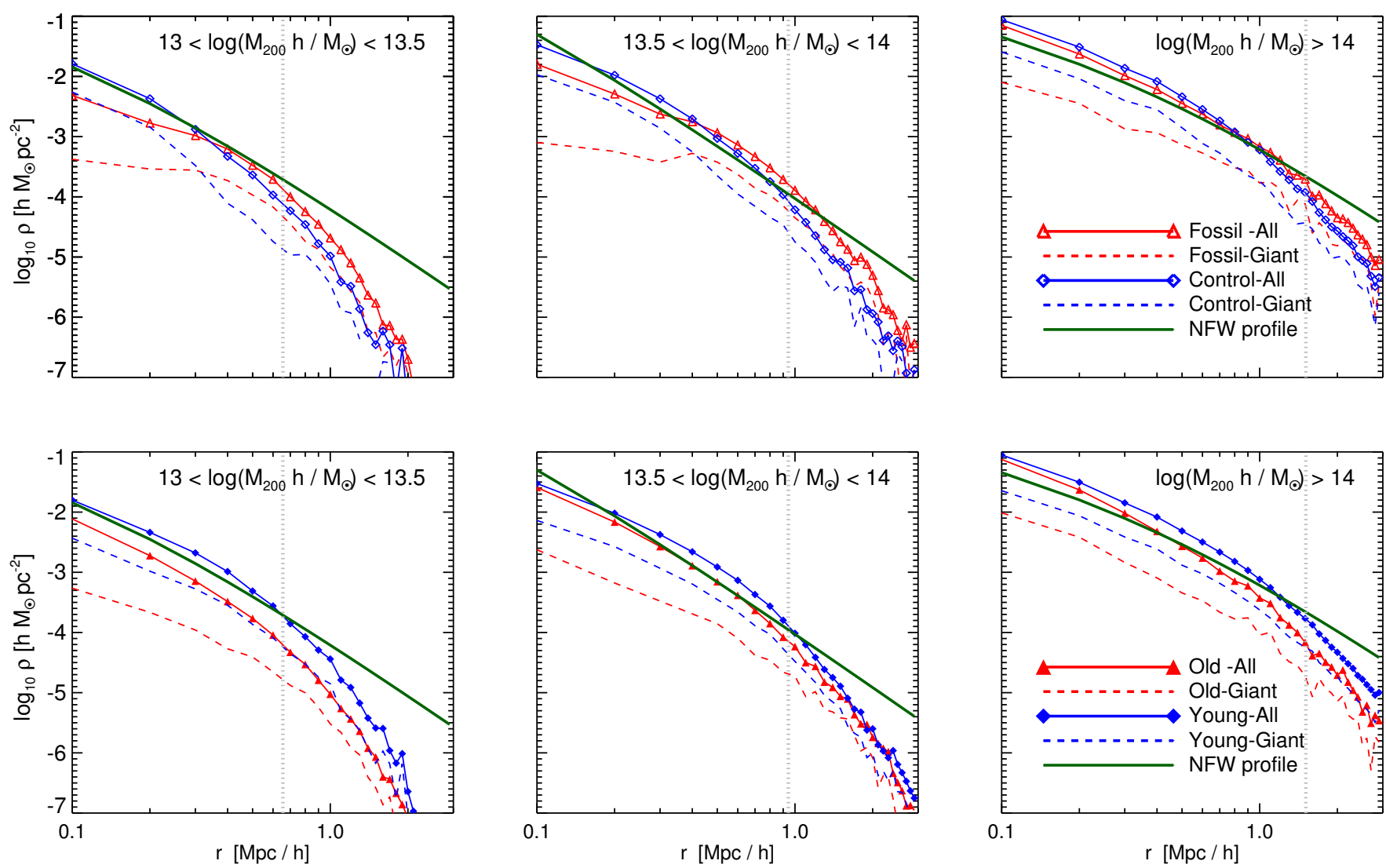

FIG. 4.- Group stellar mass density profiles in fossil (open red triangles) and control (open blue triangles) groups (top panels) as well as old (red triangles) and young (blue triangles) groups of galaxies (bottom panels). The same distribution for giant galaxies in Fossil (dashed red line), control (dashed blue line) groups as well as old (dashed red line) and young (dashed blue line) groups are shown in the top panels and bottom panels, respectively. The poisson error bars in each points are over 0.5 dex. The NFW profile (Navarro et al. 1996) is displayed (green line) for reference. These distributions are shown for 3 bins of $z=0$ halo mass increasing from left to right. The sharp declines of stellar mass density beyond the virial radii (grey dotted-lines) is due to the assignment of galaxies to the FoF halo in the semi-analytical model.

\subsection{Radio luminosity vs. last major merger}

In a recent study, Khosroshahi et al. (2017) used GAMA observations to show that most massive galaxies in dynamically unrelaxed galaxy groups have higher AGN accretion rates in comparison to those in dynamically relaxed galaxy groups. They argue that such an observed phenomenon is due to the presence of higher jet power in radio wavebands from the brightest galaxies. These findings also agree with our recent study of galaxy groups in the Illustris hydrodynamical simulation, in which we found a lower rate of black hole accretion at a given stellar mass of old BGGs in comparison to BGGs in young galaxy groups (Raouf, Khosroshahi \& Dariush 2016).

Here, we study how radio luminosity in dynamically old and young groups varies with stellar mass and other quantities using the RADIO-SAGE semi-analytic model. The top panel of Figure 5 shows their distribution of halo masses. In the bottom panel, we show that the younger systems $(\sim 70 \%)$ are in fact more radio-loud (by $\approx 0.4$ dex $)$ than the older systems $(\sim 57 \%)$ in this model.

So radio luminosity is enhanced in groups that have assembled (merged) recently. To understand how this impacts the BGGs, we compare, in Figure 6, the radio loudness of BGGs in young and old groups as a function of the time since the last major merger (hereafter TMM, ratio of stellar masses from $1: 1$ to $0.3: 1$ ) suffered by the BGG. The figure indicates that the radio luminosity of BGGs in young groups is enhanced by a factor 2 relative to BGGs in old groups of the same time since the last major merger. Moreover, one sees that radio loudness increases slightly with increasing TMM. This result seems surprising, given that, in RADIO-SAGE, galaxy mergers lead to gas fueling of the AGN, and then to enhanced radio power. However, as can be seen in Figure 7, it is not so much that radio luminosity increases with increasing TMM, but rather the stellar mass decreases with TMM (see the anticorrelation of stellar mass and TMM in the black contours of Figure 7, for both old and young groups).

Note that RADIO-SAGE predicts roughly similar trends of $1.4 \mathrm{GHz}$ radio luminosity versus stellar mass, separated between old and young groups, as are observed (Khosroshahi et al. 2017). Our previous analysis of the ILLUSTRIS hydrodynamic simulation has shown the higher AGN accretion rate of BGGs hosted by dynamically young groups compared to the old systems at a given BGG stellar mass (Raouf, Khosroshahi \& Dariush 2016), as confirmed with the observational study of Khosroshahi et al. (2017). Here, we show that the BGGs in late formed systems have higher $1.4 \mathrm{GHz}$ radio emission, independent of the elapsed time since its last major 

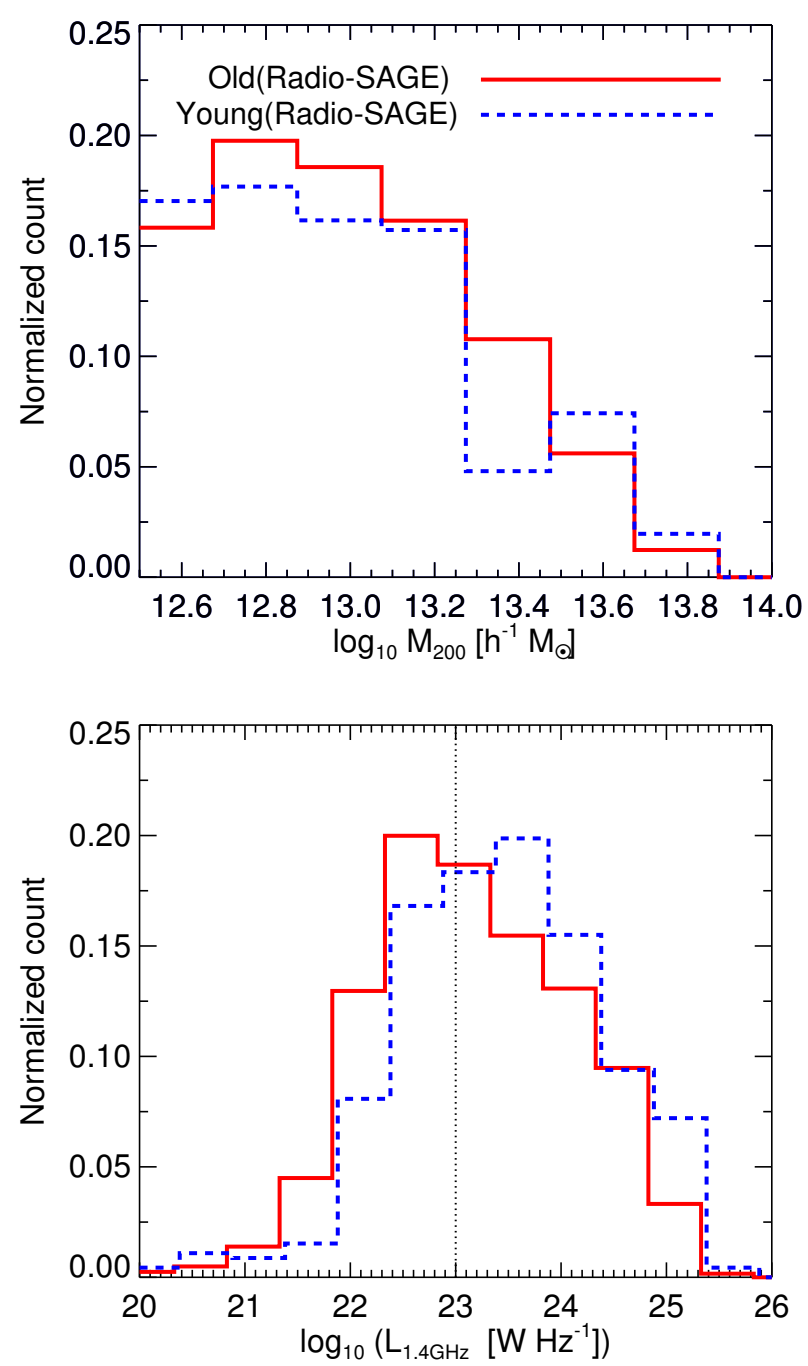

FIG. 5.- Distribution of $z=0$ halo mass (top) and $1.4 \mathrm{GHz}$ radio luminosity (bottom) for dynamically old (red line) and young (blue dashed line) galaxy groups identified at $z=0$ in the RADIO-SAGE semi-analytic model.

merger.

\section{DISCUSSION AND SUMMARY}

This paper focuses on the merger history of the brightest group galaxies in dynamically old and young galaxy groups to understand the connection between their evolution and that of their host halos. Our study is primarily limited to group size halos. We apply two semi-analytic models of galaxy formation to follow the BGGs: the Munich model (Guo et al. 2011) and the newly developed RADIO-SAGE model (Raouf et al. 2017), both run on the Millennium Simulation. In particular, RADIO-SAGE allows a more accurate analysis of the AGN activity of such galaxies. In our study, we make a distinction between fossil/control galaxy groups and dynamically old/young groups. The fossil/control distinction is driven by the luminosity gap between the two most luminous galaxies in group halo (Jones et al. 2003), while the old/young is based on the mass assembly history of the halo (Raouf et al. 2014). It has been previously shown that a large fraction of old/young galaxy groups are those with a

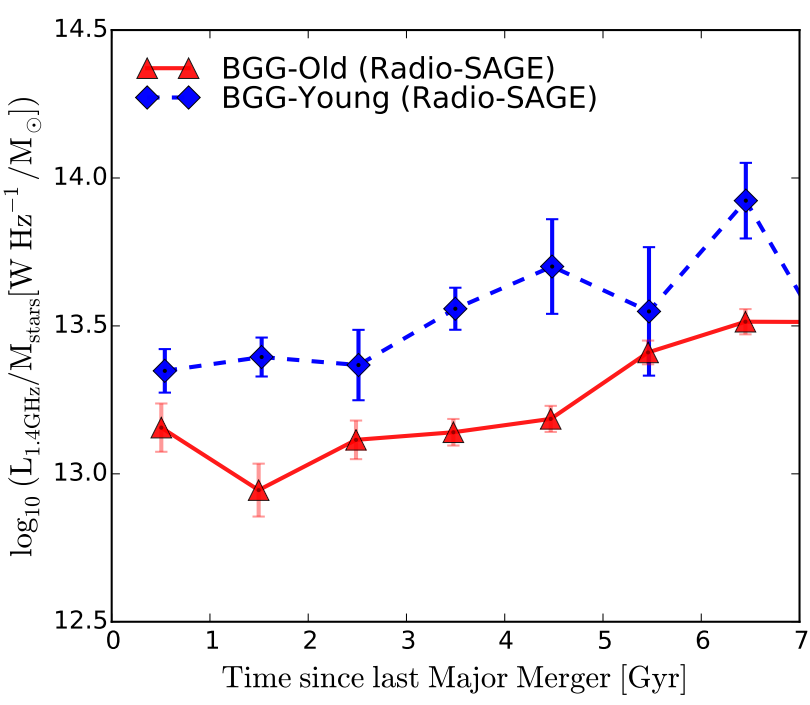

Fig. 6.- Radio loudness (luminosity at $1.4 \mathrm{GHz}$ divided by stellar mass) of BGGs at $z=0$ versus the time since the last major merger suffered by the BGG, in subsamples of groups defined by the epoch of their last major merger. The red triangles and blue diamonds show the medians and $\sigma / \sqrt{N}$ uncertainties for BGGs of old and young groups, respectively.

large/small luminosity gap, signaling some similarities in the evolution of fossil groups and dynamically old groups.

We find that the BGG major merger phenomenon is an earlier event in relaxed groups than in unrelaxed groups. Furthermore, the last major merger of BGGs hosted in young groups occurs at a later epoch than the same process in fossil and old groups. This is, of course, expected, since galaxy mergers involve subhalos, that also build up the halos. Statistically, more than $85 \%$ of young groups have experienced their last major merger before a lookback time of $1 \mathrm{Gyr}$, in comparison to less than $55 \%$ for old groups. The BGG in old and fossil systems have a lack of recent major mergers in their evolution, in agreement with earlier observations of fossil groups of galaxies (Khosroshahi et al. 2006b; Smith et al. 2010). This was also shown by Von Benda-Beckmann et al. (2008) who highlight of the same results but with limited statistics. Our results are also in agreement with the recent study of Kundert, D'Onghia \& Aguerri (2017) using the Illustris simulations and the assembly history of high luminosity gap groups.

In the $\Lambda \mathrm{CDM}$ cosmological framework, one expects that the group velocity dispersion should be roughly constant since $z=1$. The evolution of the velocity dispersion is mainly driven by the evolution of the virial velocity, with radial anisotropy playing a small role. Such an evolution with redshift is affected by mass assembly, halo concentration, and the expansion rate of the Universe.

We obtain interesting results when tracing the group velocity dispersion with different galaxy populations in different classes of groups. We find that the velocity dispersion of old groups traced by very luminous galaxies decreases in time, except for the most massive groups. This is a sign of dynamical friction causing the massive galaxies to dissipate their orbital energy. Since the dy- 

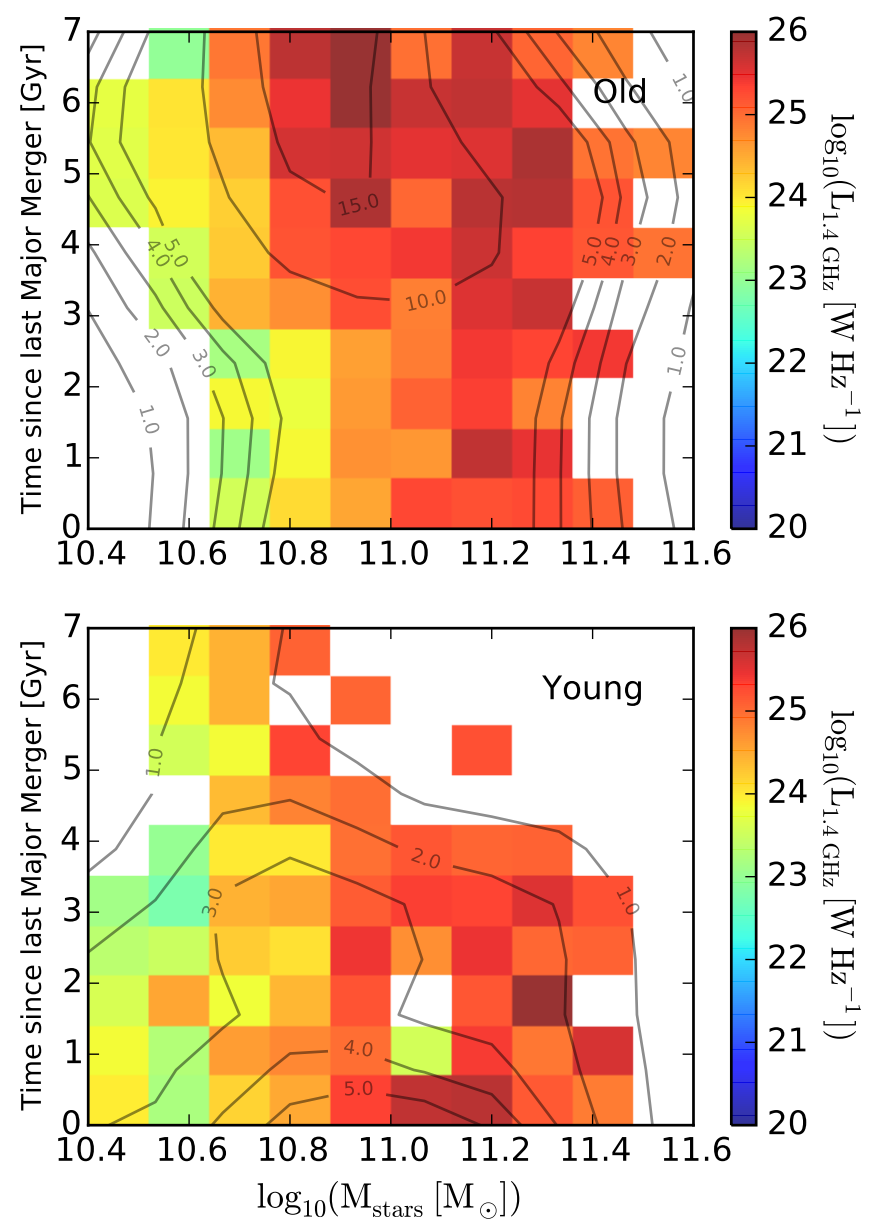

FIG. 7.- Time since the last major merger suffered by the BGGs versus their stellar mass for the BGGs of old (top panel) and young (bottom panel) groups, with symbols color-coded by the $1.4 \mathrm{GHz}$ radio log luminosity, as derived from the RADIO-SAGE SAM. The black contours show the density of galaxies per pixel (after smoothing by a Gaussian with $\sigma=1.0$ pixel).

namical friction time scales as the dynamical time (which depends on position within the virial sphere, but not on group mass), times a roughly linear function of the ratio of group mass to galaxy mass, it is too long in the high-mass groups to cause a decrease in their velocity dispersion traced by these giant galaxies. Interestingly, we see this effect of dynamical friction in low-mass groups traced by intermediate luminosity galaxies, which, again is caused by the short dynamical friction time expected for such galaxies.

Another interesting feature is the slight rise of the velocity dispersion of young groups of intermediate and high masses. These young groups are acquiring their mass more rapidly than the average group, and thus their virial velocity is also rising instead of remaining roughly constant, as is the case for normal groups.

It is important to note that higher fractions of BGG counterparts (giant galaxies) hosted in old systems are located in the central regions $(r \sim 0.2 \mathrm{Mpc}$ from the group center). This, in turn, shows the effect of an offset between the BGG and group center when identifying relaxed and unrelaxed systems.

We use the RADIO-SAGE semi-analytic model of galaxy formation, perhaps the only such model offering an observable related to AGN outflows, to compare the relationship between the activity of the central supermassive black hole as quantified from the $1.4 \mathrm{GHz}$ radio loudness of the BGG and the epoch of last major merger in central dominant galaxies hosted by dynamically old and young galaxy groups. By considering the results of the recent observational study of Khosroshahi et al. (2017) showing the higher accretion rate of BGGs in unrelaxed systems, we suggest that BGGs in groups that formed recently have higher radio luminosity (for the same stellar mass), independent of the elapsed time since its last major merger.

Extensions to our current work will focus on the stellar populations of relaxed and unrelaxed galaxy groups using both observations and cosmological simulations.

\section{ACKNOWLEDGMENTS}

We thank the anonymous referee for their constructive comments and suggestions which helped to improve the paper. The Radio Semi-Analytic Galaxy Evolution (Radio-SAGE) model used in this study is publicly available for download at https://github.com/mojtabaraouf/sage as subgroup of main SAGE repository. The Millennium Simulation databases used in this paper, and the web application providing online access to them, were constructed as part of the German Astrophysical Virtual Observatory (GAVO): http://www.mpagarching.mpg.de/millennium. We acknowledge the Virgo Consortium for access to this data.

\section{APPENDIX}

\section{VELOCITY DISPERSION MODEL FOR AN ISOLATED HALO}

This section describes how to estimate the velocity dispersion of an isolated halo. We begin by computing the evolution of the circular velocity at the virial radius, $v_{\mathrm{v}}$, hereafter virial velocity. Given the definition of the virial radius, the virial mass enclosed within it is

$$
M_{\mathrm{v}}(z)=\frac{\Delta}{2} \frac{H^{2}(z) r_{\mathrm{v}}^{3}}{G},
$$

where $G$ is the the gravitational constant and $\Delta=200$ here is the mass overdensity relative to the critical density of the Universe at the virial radius. Since the circular velocity at the virial radius satisfies $V_{\mathrm{v}}^{2}=G M_{\mathrm{v}} / r_{\mathrm{v}}$, one obtains with equation (A1)

$$
V_{\mathrm{v}}^{3}=\sqrt{\frac{\Delta}{2}} G M_{\mathrm{v}}(z) H(z) .
$$


We adopt the characteristic halo mass evolution of $M_{\mathrm{v}}(z)=M_{0} \exp [-z /(1+z)]$ (Wechsler et al. 2002) and the canonical evolution of the Hubble constant for a flat Universe with $H(z)=H_{0} E(z)$, with $E(z)=\sqrt{\Omega_{\mathrm{m}}^{0}(1+z)^{3}+1-\Omega_{\mathrm{m}}^{0}}$, where $\Omega_{\mathrm{m}}^{0}$ is the present-day matter density parameter. Note that the product $E(z) M(z)$ varies by less than $\pm 2 \%$ for $1>z>0$, and hence the virial velocity is roughly constant since $z=1$. This was previously noted from the evolution of halos obtained with Monte Carlo halo merger trees (Mamon et al. 2012).

Since halos are well fit by NFW density profile (Navarro, Frenk \& White 1996), the velocity dispersion can be simply expressed in terms of $V_{\mathrm{v}}$. According to figure 7 of tokas \& Mamon (2001), the ratio of aperture velocity dispersion (averaged over a cylinder) to circular velocity at the virial radius is of order 0.6 to 0.7 , depending on halo concentration and velocity anisotropy. For isotropic orbits, this ratio is between 0.62 and 0.66 for reasonable concentrations (Mauduit \& Mamon 2007). For more realistic orbits that are isotropic in the center and become gradually mildly radial at large radii, this ratio is between 0.65 and 0.69 for reasonable concentrations (Mamon, Biviano \& Boué 2013). In Figure 3 the expected $1 \mathrm{D}$ velocity dispersion is shown as the black line, with typical 0.25 dex uncertainties in halo mass highlighted as the blue shaded region assuming that the ratio of aperture velocity dispersion to circular velocity at the virial radius is 0.65 .

\section{REFERENCES}

Aguerri, J. A. L., Girardi, M., Boschin, W., et al. 2011, A\&A, 527, A143

Ahn, C.P., Alexandroff, R., Prieto, C.A. et al., ApJS, 211, 17

Barnes, J.E., 1989, Nature, 338, 123

Bower, R.G., Benson, A.J., Malbon, R., et al., 2006, MNRAS, 370,645

Buote, D. A. 2016, arXiv:1611.08600

Bullock, J. S., Kolatt, T. S., Sigad, Y., et al. 2001, MNRAS, 321, 559

Chandrasekhar, S., ApJ, 97, 255

Cole, S., Lacey, C. G., Baugh, C. M., \& Frenk, C. S., 2000 MNRAS, 319, 168

Cole, S., Helly, J., Frenk, C. S., \& Parkinson, H., 2007, MNRAS, 383,546

Croton, D.J., Springel, V., White, S.D. et al., 2006, MNRAS, 365, 11

Croton, D. J. and Stevens, A. R. H., 2016, ApJS, 222, 22

Cui, W., Springel, V., Yang, X., De Lucia, G., \& Borgani, S., 2011, MNRAS, 416(4), 2997

Cypriano, E. S., De Oliveira, C. L. M., \& Sodr Jr, L., 2006, AJ, 132,514

De Lucia G., Blaizot J., 2007, MNRAS, 375, 2

De Oliveira, C. L. M., Cypriano, E. S., \& Sodr Jr, L., 2006, AJ, 131, 158

Davis, M., Efstathiou, G., Frenk, C. S., White, S. D. M., 1985, ApJ, 292, 371

Dariush, A., Khosroshahi, H.G., Ponman, T.J. et al., 2007, MNRAS, 382, 433

Dariush, A. A., Raychaudhury, S., Ponman, T. J. et al., 2010, MNRAS, 405, 1873

Díaz-Giménez, E., Muriel, H., Mendes de Oliveira, C., 2008, A\&A, 490, 965

DOnghia, E., Sommer-Larsen, J., Romeo, A. D. et al., 2005, ApJ, 630, L109

Driver, S.P., Hill, D.T., Kelvin, L.S. et al., 2011, MNRAS, 413(2), 971

Farhang, A., Khosroshahi, H. G., Mamon, G. A., Dariush, A. A., \& Raouf, M. 2017, ApJ, 840, 58

Gozaliasl, G., Khosroshahi, H. G., Dariush, A. A. et al., 2014, A\&A, 571, A49

Gozaliasl, G., Finoguenov, A., Khosroshahi, H. G., et al. 2016, MNRAS, 458, 2762

Guo, Q., White, S., Boylan-Kolchin, M. et al., 2011, MNRAS, 413, 101

Guo, Q., \& White, S. D. M. 2008, MNRAS, 384, 2

Hickson, P. 1982, ApJ, 255, 382

Hopkins, P. F., Bundy, K., Croton, D., et al. 2010, ApJ, 715, 202

Jetha, N. N., Hardcastle, M. J., Babul, A. et al., 2008, MNRAS, 384,1344

Jetha, N. N., Khosroshahi, H., Raychaudhury, S., Sengupta, C., Hardcastle, M., 2009, in The Monster's Fiery Breath: Feedback in Galaxies, Groups, and Clusters, AIP Conf. Proc., vol 1201, p. 305, arXiv:0909.0221

Jiang, C. Y., Jing, Y.-P., Faltenbacher, A., Lin, W. P., Li, C., 2008, ApJ, 675, 1095
Jones, L. R., Ponman, T. J., \& Forbes, D. A., 2000, MNRAS, 312(1), 139

Jones, L.R., Ponman, T.J., Horton, A. et al., 2003, MNRAS, 343, 627

Khosroshahi, H. G., Jones, L. R., Ponman, T. J., 2004a, MNRAS, 349,1240

Khosroshahi, H. G., Raychaudhury, S., Ponman, T. J., Miles, T. A., Forbes, D. A., 2004b, MNRAS, 349, 527

Khosroshahi, H. G., Maughan, B. J., Ponman, T. J., Jones, L. R., 2006a, MNRAS, 369, 1211

Khosroshahi, H. G., Ponman, T. J., Jones, L. R., 2006b, MNRAS, 372, L68

Khosroshahi, H. G., Ponman, T. J., Jones, L. R., 2007, MNRAS, 377,595

Khosroshahi, H. G., Gozaliasl, G., Rasmussen, J. et al., 2014, MNRAS, 443, 318

Khosroshahi, H. G., Raouf, M., Miraghaei, H., et al. 2017, arXiv:1704.09029

Komatsu, E., Kogut, A., Nolta, M. R. et al., 2003, ApJS, 148, 119

, Krivitsky, D. S. \& Kontorovich, V. M., 1997, A\&A, 327, 921

Kundert, A., D’Onghia, E., \& Aguerri, J. A. L. 2017, arXiv: 1706.08542

Kundert, A., Gastaldello, F., D'Onghia, E. et al., 2015, MNRAS, 454(1), 161-176

La Barbera, F., de Carvalho, R. R., de la Rosa, I. G., et al. 2009, AJ, 137, 3942

Liske, J., Baldry, I.K., Driver, S.P. et al., 2015, MNRAS, 452(2), pp.2087-2126.

Łokas, E. L. \& Mamon, G. A., 2001, MNRAS, 321, 155

Makino, J. \& Hut, P., 1997, ApJ, 481, 83

Mamon, G. A. 1987, ApJ, 321, 622

Mamon, G. A. 1992, ApJ, 401, L3

Mamon, G. A., Biviano, A., \& Boué, G., 2013, MNRAS, 429, 3079

Mamon, G. A., \& Łokas, E. L. 2005, MNRAS, 363, 705

Mamon, G. A., Tweed, D., Thuan, T. X. \& Cattaneo, A., 2012, in Polychronis P., Recchi S., Hensler G., eds., Astrophysics and Space Science Proceedings Vol. 28, Dwarf Galaxies: Keys to Galaxy Formation and Evolution. p. 39 (arXiv:1103.5349), doi:10.1007/978-3-642-22018-0_3

Mauduit, J.-C. \& Mamon, G. A., 2007, A\&A, 475, 169

Miles, T. A., Raychaudhury, S., Forbes, D. A. et al., 2004, MNRAS, 355(3), 785

Milosavljevic', M., Miller, C. J., Furlanetto, S. R. \& Cooray, A.,2006, ApJ, 637, L9

Miraghaei, H., Khosroshahi, H. G., Klckner, H. R. et al., 2014, MNRAS, 444(1), 651-666.

Miraghaei, H., Khosroshahi, H. G., Sengupta, C., et al. 2015, AJ, 150,196

Naab, T., Johansson, P. H., \& Ostriker, J. P., 2009, ApJL, 699, L178

Navarro, J. F., Frenk, C. S., \& White, S. D. M., 1996, ApJ, 462, 563

Ostriker J. P., Tremaine S. D., 1975, ApJ, 202, L113

Parkinson, H., Cole, S., \& Helly, J., 2008, MNRAS, 383, 557

Ponman, T. J., Allan, D. J., Jones, L. R., Merrifield, M. \& MacHardy, I.M., 1994, Nature, 369, 462 
Prada, F., Klypin, A. A., Cuesta, A. J., Betancort-Rijo, J. E., \& Primack, J. 2012, MNRAS, 423, 3018

Raouf, M., Khosroshahi, H.G., Ponman, T. J. et al., 2014, MNRAS, 442. 1578

Raouf, M., Khosroshahi, H.G., \& Dariush, A. 2016, ApJ, 824, 140

Raouf, M., Shabala, S. S., Croton, D. J., Khosroshahi, H. G., \& Bernyk, M. 2017, arXiv:1706.06595

Robotham, A.S., Norberg, P., Driver, S.P. et al., 2011,MNRAS, 416(4), 2640

Rodriguez-Gomez, V., Genel, S., Vogelsberger, M., et al. 2015, MNRAS, 449, 49

Sales, L. V., Navarro, J. F., Lambas, D. G., White, S. D. M. \& Croton,D.J., 2007, MNRAS, 382, 1901

Schneider, D. P., \& Gunn, J. E. 1982, ApJ, 263, 14

Smith, G. P., Khosroshahi, H. G., Dariush, A. et al., 2010, MNRAS. 409.169S

Springel, V., White, S. D. M., Tormen, G. \& Kauffmann, G., 2001, MNRAS, 328, 726

Springel, V., White, S. D., Jenkins, A. et al., 2005, Nature, 435, 629
Tempel, E., Tamm, A., Gramann, M. et al., 2014, A \& A, 566, 1 Tollet, É., Cattaneo, A., Mamon, G. A., Moutard, T. \& van den Bosch, F. C. 2017, MNRAS, 471, 4170

Toomre, A. \& Toomre, J., 1972, ApJ, 178, 623

Trevisan, M., Mamon, G. A., \& Khosroshahi, H. G. 2017, MNRAS, 464, 4593

Tweed, D., Mamon, G, A., Thuan, T. X., Cattaneo, A., Dekel, A., Menci, N., Calura, F., and Silk, J., 2018, MNRAS, in press, arXiv:1802.09530

Wechsler, R. H., Bullock, J. S., Primack, J. R., Kravtsov, A. V., \& Dekel, A. 2002, ApJ, 568, 52

White, S. D. M. 1976, MNRAS, 174, 19

Van Den Bosch, F. C., Yang, X., Mo, H. J. et al., 2007, MNRAS, 376,841

Von Benda-Beckmann, A. M., DOnghia, E., Gottlo ber, S. et al., 2008, MNRAS, 386,2345 